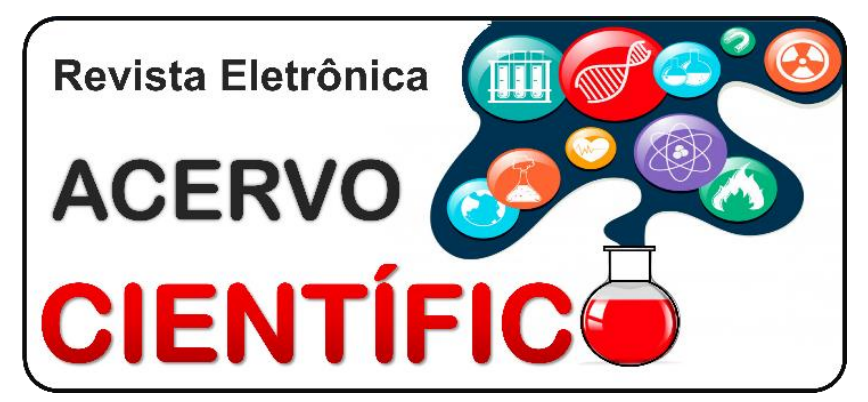

ARTIGO ORIGINAL

Recebido em: 12/2020

Aceito em: $12 / 2020$

Publicado em: 2/2021

\title{
Riscos cardiovasculares, aptidão física dos policiais militares de Anápolis
}

\author{
Cardiovascular risks, physical fitness of military policies of Anápolis
}

Riesgos cardiovasculares, aptitud física de las políticas militares de Anápolis

Patrícia Espíndola Mota Venâncio ${ }^{1 *}$, Daniel de Lima Silva1, João Pedro Diniz e Castro', Lavynia Luiza Saturnino de Melo ${ }^{1}$, Thairyne Pereira de Almeida David ${ }^{1}$, Jairo Teixeira Junior ${ }^{1,2}$, Cristina Gomes de Oliveira Teixeira ${ }^{3}$.

\begin{abstract}
Resumo: Esse artigo buscou avaliar e correlacionar os níveis de riscos cardiovasculares $(R C)$ e aptidão física (AF) dos policiais militares da cidade de Anápolis, em Goiás. Fizeram parte da amostra 40 policiais masculinos. Foram avaliados RC, pressão arterial, circunferência de cintura, IMC. Para a AF foram avaliados $\mathrm{VO}_{2 \mathrm{MÁx}}$, força muscular, flexibilidade e resistência muscular. Os resultados mostraram para a PAD $62,5 \%$ estavam classificados em níveis normais; na RCE, $70 \%$ possuem riscos para a saúde; no $\mathrm{VO}_{2 \mathrm{MÁx}}$, que $55 \%$ estavam em nível regular. Quanto aos resultados para AF, a maioria se encontra na classificação "excelente" sendo $85 \%$ na flexibilidade; $87,5 \%$ força; e $67,5 \%$, resistência. Houve correlação entre a variável $\mathrm{VO}_{2 M A ́ x}$ com a variável força, em que aqueles que obtiveram melhor $\mathrm{VO}_{2 M A ́ x}$ apresentaram maiores índices de força e a variável IMC com a variável resistência, no qual, quanto mais alto o IMC, menor foi a resistência do indivíduo. Conclui-se que a maioria dos policiais apresentaram riscos cardiovasculares e classificação excelente para aptidão física. Uma correlação encontrada foi que aqueles que possuem maior índice de força apresentaram maior $\mathrm{VO}_{2 M A ́ x}$ e os que possuem o IMC com melhores índices apresentaram melhores índices de resistência muscular.
\end{abstract}

Palavras-chave: Doenças cardiovasculares, Aptidão física, Polícia.

\begin{abstract}
This article sought to assess and correlate the levels of cardiovascular risks (CR) and physical fitness (PA) of military police officers in the city of Anápolis, Goiás. The sample included 40 male police officers. CR, blood pressure, waist circumference, BMI were evaluated. For PA, VO2MAX, muscle strength, flexibility and muscle endurance were evaluated. The results showed for PAD $62.5 \%$ were classified in normal levels; in the CER, 70\% have health risks; in VO2MÁX, that 55\% were at a regular level. As for the results for PA, most are classified as "excellent" with $85 \%$ in flexibility; $87.5 \%$ strength; and $67.5 \%$, resistance. There was a correlation between the VO2MÁX variable and the strength variable, in which those who obtained better VO2MÁX had higher strength indices and the BMI variable with the resistance variable, in which, the higher the BMI, the lower the individual's resistance. It was concluded that the majority of police officers had cardiovascular risks and an excellent classification for physical fitness. A correlation found was that those who have a higher strength index had a higher VO2Max and those with a BMI with better indexes had better muscle resistance indexes.
\end{abstract}

Keyword: Cardiovascular diseases, Physical fitness, Police.

${ }^{1}$ Centro Universitário (UniEVANGÉLICA), Anápolis - GO. *E-mail: venanciopatricia@hotmail.com

2Escola Superior de Educação Física do Estado de Goiás (Eseffego), Anápolis - GO.

3Instituto Federal de Educação, Ciência e Tecnologia de Goiás, Anápolis - GO. 
Resumen: Este artículo buscó evaluar y correlacionar los niveles de riesgo cardiovascular (RC) y aptitud física (AF) de policías militares de la ciudad de Anápolis, Goiás, con una muestra de 40 policías varones. Se evaluó RC, presión arterial, circunferencia de la cintura, IMC. Para PA, VO2MAX, se evaluaron la fuerza muscular, la flexibilidad y la resistencia muscular. Los resultados mostrados para PAD 62,5\% se clasificaron en niveles normales; en el CER, el 70\% tiene riesgos para la salud; en VO2MÁX, ese $55 \%$ estaba en un nivel regular. En cuanto a los resultados de AF, la mayoría se clasifican como "excelentes" con un $85 \%$ de flexibilidad; $87,5 \%$ de fuerza; y $67,5 \%$, resistencia. Hubo correlación entre la variable VO2MÁX y la variable fuerza, en la que los que obtuvieron mejor VO2MÁX tuvieron índices de fuerza más altos y la variable IMC con la variable resistencia, en la que a mayor IMC, menor resistencia del individuo. Se concluyó que la mayoría de los policías presentaban riesgo cardiovascular y una excelente clasificación de aptitud física. Una correlación encontrada fue que aquellos que tienen un índice de fuerza más alto tenían un VO2Max más alto y aquellos con un IMC con mejores índices tenían mejores índices de resistencia muscular.

Palabras clave: Riesgo cardiovascular, Aptitud física, Policía.

\section{INTRODUÇÃO}

Nos dias atuais a segurança pública é um dos assuntos tratados com mais importância pela sociedade, gerando uma grande expectativa em cima dos policiais militares que garantem essa segurança (MAZARIOLLI AS e RABELO I, 2019). Com o aumento das ocorrências de violência e de crime na sociedade foi criada, em 2013 a Companhia de Policiamento Especializado (CPE), que trabalha em conjunto com a Polícia Militar sendo implantada em locais estratégicos para o combate à criminalidade (MARINS EF, et al., 2019).

Com a exigência da rotina de trabalho é importante que os policiais apresentem uma boa aptidão física para que possam realizar as tarefas com mais eficiência, mais produtividade e, por consequência, diminuir o risco de acidentes (BORGES AM, et al., 2019).

A pressão do trabalho pode desencadear algumas doenças, como as crônicas não transmissíveis, principal causa de morbimortalidade no mundo. Entre elas, estão as doenças cardiovasculares (DCV), que, com o passar dos anos, tornaram-se comuns entre a população e, em especial, nos indivíduos que sofrem estresse no trabalho (PRADO CEP, 2016).

A rotina maçante de trabalho pode gerar problemas físicos e mentais, suscitando fatores de riscos que estão relacionados às DCV e déficits cognitivos como a memória, a atenção, a obesidade. O baixo nível na prática de exercício físico são alguns desses fatores condicionantes para o desenvolvimento dessas doenças (MINAYO MCS, et al., 2011).

Nesse sentido é de suma importância para a prevenção dessas doenças a prática esportiva ou a atividade física que devem ser praticadas diariamente; pois, além de trazer benefícios cardiovasculares, podem proporcionar melhoras nas diferentes áreas da saúde como memória e atenção, aptidão física e na qualidade de vida dos praticantes (RIBEIRO AG, et al., 2012).

Diante das informações apresentadas, o estudo traz a seguinte problemática: Como se encontram os riscos cardiovasculares, a aptidão física, dos indivíduos que lidam com a segurança pública? Como justificativa para a pesquisa destaca-se a importância de identificar os níveis de riscos cardiovasculares, aptidão física, para que os policiais possam ser mais bem orientados consigam melhorar seus níveis de saúde, consequentemente atingindo melhores rendimentos e qualidade no trabalho. Portanto, o presente estudo teve como avaliar e correlacionar os níveis de riscos cardiovasculares (RC) e aptidão física (AF) dos policiais militares da cidade de Anápolis, em Goiás.

\section{MÉTODOS}

Trata-se de um estudo observacional transversal de cunho quantitativo e de amostragem por conveniência realizada com os policiais militares da cidade de Anápolis, em Goiás. O estudo foi composto por 44 policiais que estavam de serviço, sendo feitos os testes antes de começarem suas atividades profissionais. 
Para participar da pesquisa os policiais não poderiam apresentar problema físico que viesse comprometer a realização dos testes. Foram excluídos da pesquisa aqueles com testes físicos e/ou questionários incompletos, além dos que estavam de férias.

Como instrumentos e procedimentos para os riscos cardiovasculares foram avaliados pressão arterial, circunferência de cintura, IMC, Cortisol e proteína $C$ reativa. A pressão arterial foi aferida por meio do aparelho de pressão digital G-Tech MA100 (Shenzhen, China), e realizada com os participantes sentados antes de serem submetidos a qualquer tipo de teste de esforço, e, para classificação, foi utilizada a tabela de referência da Sociedade Brasileira de Cardiologia (SBC) (MALACHIAS MVB, 2016).

A Circunferência de cintura e o IMC foram coletados em conjunto, aferindo-se a estatura do participante e o peso com a balança digital S Sanny com estadiômetro - BL201PP (Porto Alegre, Brasil) para cálculo do IMC (massa corpora/ altura²) (WHO, 2000).

Para a circunferência de cintura e a circunferência de abdômen foi empregada uma trena antropométrica Sanny TR4010 (Porto Alegre, Brasil), sendo utilizadas as tabelas de referência da OMS para a classificação do IMC e da CC, respectivamente (OLIVEIRA LF e RODRIGUES PAS, 2016).

A Composição Corporal por meio de medidas antropométricas foi executada antes dos testes de esforço. Utilizou-se o protocolo de três dobras (tricipital, supra ilíaca e coxa medial), sendo cada dobra aferida três vezes e sendo utilizada a média entre elas.

A relação cintura-estatura $(R C E)$ foi aferida com a fita métrica na altura do umbigo, e, para o cálculo da RCE, utilizou-se a medida da circunferência de cintura (CC) dividida pela estatura ambas em centímetros com resultado variando de valores próximos de zero (0) a um (1). Quanto aos resultados foi padronizado como ponto de corte o valor de 0,5 , portanto valores iguais ou superiores a este parâmetro indicariam risco aumentado de doenças cardiovasculares.

A coleta de sangue foi realizada por uma biomédica que se deslocou ao batalhão para as coletas em seguida foi levado ao um laboratório Diagnóstico para análise. O sangue foi coletado em apenas um momento e o volume de $20 \mathrm{~mL}$ para serem distribuídos nas dosagens do lipidograma (coleta $5 \mathrm{~mL}$ para análise de $1 \mathrm{~mL}$ de soro), glicemia de jejum (coleta $4 \mathrm{~mL}$ para análise de $1 \mathrm{~mL}$ de plasma), proteína C-reativa ultrassensível (coleta $5 \mathrm{~mL}$ para análise de $1 \mathrm{~mL}$ de soro) e cortisol (coleta $5 \mathrm{~mL}$ para análise de $1 \mathrm{~mL}$ de soro). As amostras foram transportadas para laboratório para serem centrifugadas e preparadas para dosagem dos parâmetros. Todo material da coleta (seringas, agulhas e sistema a vácuo) foi descartado em recipiente específico para perfuro cortante (Marca descarpack, amarelo pardo, São Paulo, Brasil). Para o cortisol foi utilizado o método Quimioluminescência e para a proteína C reativa o método Imunoturbidimetria.

Com o intuito de avaliar a aptidão física foram feitos os testes do banco de Wells para a flexibilidade, abdominais para resistência muscular localizada, teste de flexão de braço para força de membros superiores (MMSS), teste de léger para avaliação do $\mathrm{VO}_{2 M A ́ x} \mathrm{e}$, para composição corporal, o protocolo de três dobras cutâneas. Para o Teste de flexibilidade, por meio do banco de Wells, foi utilizado o protocolo padrão de avaliação em que o avaliado possui duas chances e é considerado o maior score. Foi utilizado o banco de Wells Instant Flex Sanny BW2002, Porto Alegre, Brasil.

O Teste de resistência por abdominais consiste em o avaliado realizar o número máximo de abdominais dentro deum minuto, usando a tabela de referência de acordo com a idade e o número de abdominais realizado para saber sua classificação. Para a marcação do tempo foi utilizado o cronômetro da marca Finis $3 \mathrm{x}-300 \mathrm{M}$

Para o Teste de força, por meio de flexão de braços, o avaliado teve que realizar até a falha o maior número de repetições de flexão de braço (apoio ao solo) sem tempo determinado, para que seja classificado pela tabela de referência, levando em conta a idade e o número de repetições executadas.

No Teste de $\mathrm{VO}_{2 \mathrm{MAx}}$, por meio do teste de Léger, os avaliados foram colocados em grupos de cinco pessoas. O teste consistiu na utilização de uma pista de $20 \mathrm{~m}$ em que, a cada toque de sinal sonoro, o avaliado deveria cruzar uma das extremidades da pista, sendo que, em cada estágio, o intervalo entre um 
toque e outro diminuiria e o avaliado consequentemente deveria correr mais rápido para cruzar a extremidade. Para classificação foram utilizados a fórmula e a tabela padrão do protocolo.

Os dados foram coletados do dia 17 ao dia 19 de dezembro de 2019 , das 8 h às $12 \mathrm{~h}$, no batalhão da corporação. Os primeiros procedimentos foram a coleta de sangue, logo em seguida a aferição da PA e coleta de dados antropométricos. Depois foram realizados os testes os testes físicos. Após a coleta de todos os dados os resultados foram tabulados no programa Microsoft Excel 2019.

A análise dos dados foi por média, desvio-padrão. A normalidade dos dados foi feita pelo teste ShapiroWilk. Foi feito um teste de Kuskal-Wallis com post hoc de Dunn (distribuição assimétrica) e uma correlação de sperman para as variáveis risco cardiovascular, memória, atenção, qualidade de vida. $O$ valor de $p$ considerado foi $<0,05$. Os dados foram analisados na Software Statistica Package for Social Science (SPSS 20.0). O estudo foi aprovado pelo Comitê da UniEVANGÉLICA sob o no 4.080.786.

\section{RESULTADOS}

A amostra estudada foi de 40 avaliados, dentre eles 25 estavam em níveis normais, seis eram limítrofes, sete com hipertensão nível 1, e cinco tinham hipertensão nível 2, conforme a mensuração da PAD. De acordo com a RCE, 28 policiais possuíam riscos para saúde e 12, não apontavam riscos para saúde. 0 teste de $\mathrm{VO}_{2 \text { м́x }}$ mostrou que 22 estavam em nível regular. Os resultados para flexibilidade, força e resistência, respectivamente, apresentaram classificações de $85 \%, 87,5 \%$ e $67,5 \%$ para "excelente". de acordo com a Tabela 1.

Tabela 1 - Classificação geral das variáveis.

\begin{tabular}{|c|c|c|c|c|c|c|}
\hline \multicolumn{7}{|c|}{ Riscos cardiovasculares n (\%) } \\
\hline \multirow{2}{*}{ PAD } & \multirow{2}{*}{$\begin{array}{l}\text { Normal } \\
25(62,5)\end{array}$} & \multirow{2}{*}{$\begin{array}{c}\text { Limítrofe } \\
6(15,0)\end{array}$} & \multirow{2}{*}{$\begin{array}{c}\text { Hipertensão } 1 \\
7(17,5)\end{array}$} & \multicolumn{2}{|c|}{ Hipertensão 2} & Total \\
\hline & & & & $5(5,0)$ & & $40(100)$ \\
\hline \multirow{2}{*}{ RCE } & Com risco & & Sem risco & --- & & Total \\
\hline & $28(70,0)$ & & $12(30,0)$ & --- & & $40(100)$ \\
\hline \multicolumn{7}{|c|}{ Aptidão física n (\%) } \\
\hline \multirow{2}{*}{$\mathrm{VO}_{2 M A X}$} & Superior & Excelente & Bom & Regular & Fraco & Total \\
\hline & $1(2,5)$ & $3(7,5)$ & $12(30,0)$ & $22(55,0)$ & $2(5,0)$ & $40(100)$ \\
\hline \multirow{2}{*}{ Flexibilidade } & Excelente & Bom & Médio & Muito fraco & -- & Total \\
\hline & $34(85,0)$ & $3(7,5)$ & $2(5,0)$ & $1(2,5)$ & -- & $40(100)$ \\
\hline \multirow{2}{*}{ Força } & Excelente & -- & -- & Acima da Média & -- & Total \\
\hline & $35(87,5)$ & -- & -- & $5(12,5)$ & -- & $40(100)$ \\
\hline \multirow{2}{*}{ Resistência } & Excelente & \multicolumn{2}{|c|}{ Acima da Média } & Média & -- & Total \\
\hline & $27(67,5)$ & \multicolumn{2}{|c|}{$6(15,0)$} & $7(17,5)$ & -- & $40(100)$ \\
\hline
\end{tabular}

Fonte: Venâncio PEM, et al., 2020.

A Tabela 2 mostra os resultados quanto à comparação da pressão arterial diastólica, à relação cintura estatura e à memória entre os indivíduos sem processo inflamatório e entre os indivíduos com processo inflamatório. Os policiais com processo inflamatório alterado obtiveram melhores índices $(78,3 \%)$ de PAD normal em relação aos que estão com processos inflamatórios normais $(41,2 \%)$. Entretanto, dos avaliados com processo inflamatório e $82,6 \%$ estão com risco cardiovascular de acordo com a relação cintura estatura. 
Tabela 2 - Comparação das variáveis de acordo com o processo inflamatório.

\begin{tabular}{ccc}
\hline & Pressão arterial diastólica \\
\hline Classificação & S/ processo inflamatório n (\%) & C/ processo inflamatório n (\%) \\
\hline Ótima & - & - \\
Normal & $7(41,2)$ & $18(78,3)$ \\
Limítrofe & $5(29,4)$ & $1(4,3)$ \\
Hipertensão 1 & $3(17,6)$ & $4(17,4)$ \\
Hipertensão 2 & $2(11,8)$ & - \\
\hline \multicolumn{3}{c}{ Relação cintura estatura } \\
\hline Classificação & S/ processo inflamatório $\mathbf{n}(\%)$ & C/ processo inflamatório n (\%) \\
\hline Com risco & $9(52,9)$ & $19(82,6)$ \\
Sem risco & $8(47,1)$ & $4(17,4)$ \\
\hline
\end{tabular}

Fonte: Venâncio PEM, et al., 2020.

A tabela a seguir apresenta os resultados das correlações entre as variáveis. Para as variáveis memória, atenção e qualidade de vida não houve correlação com o risco cardiovascular; mas houve correlação entre a variável $V_{2} O_{2 m a ́ x}$ com a variável Força, em que aqueles que obtiveram melhor $\mathrm{VO}_{2 M A ́ x}$ apresentaram maiores índices de força, e entre a variável IMC com a variável Resistência, na qual quanto mais alto o IMC menor foi a resistência do indivíduo (Tabela 3).

Tabela 3 - Correlação entre as variáveis.

\begin{tabular}{ccc}
\hline Variáveis & $\mathbf{r}$ & $\mathbf{p}$ \\
\hline $\mathrm{VO}_{2 M A ́ x} \times$ força & 0,501 & 0,001 \\
IMC $\times$ resistência & 0,400 & 0,001 \\
\hline
\end{tabular}

Fonte: Venâncio PEM, et al., 2020.

\section{DISCUSSÃO}

Wright BR, et al. (2011) realizou um estudo em que comparou um grupo de policiais com pessoas de outras atividades ocupacionais, coletando dados de diversas variáveis, entre elas a pressão arterial diastólica $(P A D)$ e a proteína C-reativa (PCr), e constataram que os níveis mais elevados de $P A D(\beta=0,16$, $P=0,04)$ estão associados à categoria ocupacional, e os níveis mais altos de $\operatorname{PCr}(\beta=0,23, P=0,01)$, ligados ao status de policial, resultados esses que agregam ao presente estudo, em que a maioria dos policiais que tinha um processo inflamatório teve a PAD em níveis mais baixos.

Entre as medidas preventivas, é notório que o exercício físico seja umas das atividades não farmacológica realizada pelo ser humano para a manutenção da saúde e bem-estar, comprovado sua eficácia tanto física, como fisiológica. A prática regular do mesmo, assim como a adoção de uma vida ativa, pode proporcionar tratamento natural e também a prevenção de uma série de doenças, dentre elas as Doenças Cardiovasculares (CICHOCKI M, et al., 2017).

Em um estudo de Damasceno RKV, et al., (2016) foram avaliados 25 policiais militares do Estado do Ceará, com o objetivo de avaliar a composição corporal e antropométrica. Foi mensurado o Índice de massa corporal (IMC), a relação cintura estatura (RCE), a relação cintura quadril (RCQ) e a circunferência de cintura (CC), tendo como resultado, através da RCE, que $15(60 \%)$ dos policiais estavam dentro do índice de risco a saúde e que $10(40 \%)$ estavam fora de risco. A CC permite uma avaliação do acúmulo de gordura na região central abdominal, e esse acúmulo tem relação direta com a deposição de tecido adiposo no interior da cavidade abdominal, fator que está associado ao aumento do risco de mortalidade (OLIVEIRA LF e RODRIGUES PAS, 2016).

Esses resultados trazem relevância para esta pesquisa, já que demonstram uma similaridade com este estudo, no qual a maioria dos policiais avaliados foram classificados com riscos à saúde e apresentam processo inflamatório alterado que pode estar relacionado com os altos níveis de RCE. Com o crescimento 
da necessidade em analisar os motivos que leva o indivíduo a adquirir uma DCV, surgem indicadores como o Índice de Massa Corpórea (IMC), Composição Corporal (CC), Relação Cintura - Quadril (RCQ), High Density Lipoproteins (HDL), Pressão Arterial Sistólica (PAS), Pressão Arterial Diastólica (PAD), níveis de glicose, níveis de VO2, entre outros. Estes indicadores são frequentemente utilizados em estudos sobre riscos cardiovasculares, uma vez que eles identificam alterações fisiológicas precisas individuais (SOUZA MAN, 2020).

As doenças cardiovasculares podem ser identificadas também pelo aumento da circunferência de cintura, que possibilita alterações metabólicas causadas pelo excesso de gordura corporal, um preditivo à DC's (PEREIRA ES e MOREIRA OC, 2011). Lima-dos-Santos AL, et al., (2018) realizaram um estudo para mensurar a Aptidão Física Relacionada a Saúde (AFRS) dos policiais militares da Paraíba, com o objetivo de comparar vários componentes da AFRS em policiais militares do Batalhão de Operações Policiais Especiais (BOPE) e do Batalhão de Policiamento de Trânsito (BPTRAN). Obteve-se correlações positivas e negativas significantes entre o consumo máximo de oxigênio $\left(V_{2} O_{2 M A x}\right)$ e as variáveis $C C, I M C, P G$ e $R M L$ $(p<0,05)$, exceto para flexibilidade no BOPE. Além de uma ótima captação de glicose, o nosso corpo precisa que a captação de oxigênio, o transporte deste até os músculos e a sua utilização seja feita com a máxima eficiência.

E o consumo máximo de oxigênio (VO2 Máximo), um dos principais parâmetros utilizados para avaliação da capacidade cardiorrespiratória em relação à entrega do oxigênio aos tecidos do corpo humano. $E$ quando isso ocorre com eficiência demonstra que o indivíduo é fisicamente ativo, com menos preocupação de disfunções fisiológicas que possam comprometer a saúde vascular, porém, uma vez que o mesmo apresente disfunção, ou seja, esta captação de oxigênio for insuficiente durante um esforço físico, o risco de o indivíduo sofrer de alguma doença como a insuficiência cardíaca é maior (CICHOCKI M et al., 2017).

O estudo de Zhang J, et al. (2019) mostrou que uma melhor aptidão física está ligada a baixos riscos dos policias apresentarem doenças cardiovasculares, corroborando com o estudo de Schilling R, et al. (2019), que avaliou 201 militares da Basileia, uma cidade na parte noroeste da Suíça, e encontrou nos resultados que altos níveis de aptidão cardiorrespiratória foram associados a um risco cardiovascular reduzido, enquanto altos níveis de estresse ocupacional foram associados a mais sintomas de estresse no trabalho e aumento do sofrimento psicológico geral. Resultados estes que podem ser reforçados no presente estudos ao correlacionar os riscos cardiovasculares e aptidão física, constatou que os policiais que apresentaram melhores os resultados da aptidão física menores foram os valores para os riscos cardiovascular.

Para prevenção dessas doenças, principalmente quando se analisa os seus fatores de riscos, é interessante que seja desenvolvido programas que influenciem o modo de vida da população (RIBEIRO, AG, et al., 2012), tornando melhor a aptidão física (AF). As respostas adquiridas no presente estudo mostram uma desigualdade nos resultados que, ao avaliar as variáveis da AFRS e realizar as correlações, duas apresentaram significância $\mathrm{VO}_{2 M A x} \mathrm{x}$ força, considerando que quem possui um melhor $\mathrm{VO}_{2 M A ́ x}$ apresenta melhores níveis de força e IMC $\mathrm{x}$ resistência em que os indivíduos que apresentaram um maior IMC tiveram um menor índice de resistência, levando a observar que os policiais que mantêm uma prática de atividade física regular apresentam uma melhor AFRS.

Silva WM, et al. (2018) ao estudarem as condições de saúde dos policiais militares atuantes em uma Companhia Independente de Polícia Militar, constatou que a maioria dos militares entrevistados não dispõe de orientação acerca dos cuidados com a saúde, da importância da prática de atividades físicas e de uma alimentação balanceada e na avaliação do IMC dos militares em questão, o sobrepeso apareceu como sendo o problema mais comum. Resultados esses que corrobora com o presente estudo em mostrar a relevância em estudar esses indivíduos que, a cada dia, encontram-se mais e mais acometidos de pressão durante o trabalho que podem estar mais propensos a doenças e lesões ocasionadas por acidentes de trabalho ou pela falta de aptidão física relacionada à saúde.

Outro estudo que reforça a relevância do presente artigo é o estudo de Rodrigues EKB e Arruda SG (2020) ao analisar os dados de mortalidade por causas externas na região norte do estado do Espírito Santo, caracterizar os óbitos por causas externas segundo as variáveis e identificar as principais causas 
específicas de morte por causas externas no DATASUS no período de 2010-2018. Constatou que as doenças cardiovasculares foram responsáveis por 362,091 mortes em 2017, somente no Brasil o que representa três em cada dez óbitos. Frente a esses resultados o presente estudo evidencia a necessidade de mais estudos e da intervenção de políticas públicas afim de investir em programas socioeducativas de forma que conscientizar esse público quantos aos riscos de doenças ocasionadas pelo pressão e carga horária de trabalho, bem como, investir em programas educativos de exercícios físicos no combate aos riscos cardiovasculares, a melhoria de qualidade de vida e nos aspectos emocionais.

\section{CONCLUSÃO}

Conclui-se que os policiais apresentaram riscos para doenças cardiovasculares relacionado a RCE, uma vez que a maioria obteve altos índices. Para a aptidão física, foram identificados bons níveis para $\mathrm{VO}_{2 \mathrm{MÁx}}$, força muscular, flexibilidade e resistência muscular. Ao comparar a classificação dos indivíduos sem processo inflamatório e os indivíduos com processo inflamatório, pôde-se concluir que aqueles que possuem processo inflamatório obtiveram resultados melhores para riscos cardiovasculares. E a correlação encontrada foi que aqueles que possuem maior índice de força apresentaram maior $\mathrm{VO}_{2 M A ́ x}$ e os que possuem o IMC com melhores índices apresentaram melhores índices de resistência muscular.

\section{REFERÊNCIAS}

1. BORGES AM, et al. A Influência do Condicionamento Físico do Policial Militar do Estado de Goiás em seu Desempenho Profissional. Rev. Bra. Mil. Ciên. 2019; 11: 08-13.

2. CICHOCKI M, et al. Atividade Física e Modulação do Risco Cardiovascular. Revista Brasileira medicina do Esporte. 2017;23(1).

3. DAMASCENO RKV, et al. Composição Corporal e Dados Antropométricos de Policiais Militares de Batalhão de Choque do Estado do Ceara. Rev. Saúde Desen. Hum. 2016; 4(2): 109-11.

4. LIMA-DOS-SANTO AL, et al. Aptidão Física Relacionada À Saúde De Policiais Militares Da Paraíba. Rev. Bras. Med. Trab, 2018;16(4):429-35.

5. MALACHIAS MVB, et al. 7ª Diretriz Brasileira de Hipertensão Arterial. Arq. Bras. Card. 2016; 107(30): 79-83.

6. MARINS EF, et al. Characterization of the Physical Fitness of Police Officers: A Systematic Review. The Journal of Strength and Conditioning Research. 2019; 33(10): 2860-74.

7. MAZARIOLLI AS, RABELO I S'A. Avaliação Cognitiva de Policiais Militares e Universitários em Medidas Padronizadas de Memória, Atenção e Inteligência. Res. Meta: Ava. 2019; 11(32): 468-494).

8. MINAYO MCS, et al. Impacto das atividades profissionais na saúde física e mental dos policiais civis e militares do Rio de Janeiro. Rev. Ciên. Saúde. 2011; 16(4): 2199-2209.

9. OLIVEIRA LF, RODRIGUES PAS. Circunferência de Cintura: Protocolos de Mensuração e Sua Aplicabilidade Prática. Rev. Nutri. Vig. Saúde. 2016;4(3):90-95.

10. PEREIRA ES; MOREIRA OC. Importância Da Aptidão Física Relacionada À Saúde E Aptidão Motora Em Crianças E Adolescentes. Rev. Bras. Pres. Fisio. Exer. 2011;7(39):309-316.

11. PRADO CEP. Estresse Ocupacional: Causas E Consequências. Rev. Bras. Med. 2016;14(3):285-9.

12. RIBEIRO AG, et al. A promoção da saúde e a prevenção integrada dos fatores de risco para doenças cardiovasculares. Rev. Ciên. Saúde. 2012; 17(1): 7-17.

13. RODRIGUES EKB, ARRUDA SG. Mortalidade Por Causas Externas Na Região Norte Do Espírito Santo, 2010 A 2018. Revista Artigos.Com. 2020; 20: 1-10.

14. SCHILLING R, et al. Does cardiorespiratory fitness moderate the association between occupational stress, cardiovascular risk, and me ntal health in police officers? Int J Environ Res Public Health. 2019;16(13).

15. SOUZA MAN. Indicadores antropométricos associados a doenças crônicas, incapacidade e mortalidade entre idosos. 2020. 103 f. Tese (Doutorado em Saúde Coletiva - Área de Concentração Epidemiologia) - Instituto René Rachou, Fundação Oswaldo Cruz, Programa de Pós-Graduação em Saúde Coletiva, Belo Horizonte, 2020.

16. WRIGHT BR, et al. Law Enforcement Officer Versus Non-Law Enforcement Officer Status as a Longitudinal Predictor of Traditional and Emerging Cardiovascular Risk Factors. J. Occup. Environ. Med. 2011; 53(7): 730-734.

17. WORLD HEALTH ORGANIZATION. Obesity: preventing and managing the global epidemic. Technical Report Series 894. 2000. 252p

18. ZHANG J, et al. Prevalence of metabolic syndrome and its risk factors among 10,348 police officers in a large city of China. Med (United States). 2019; 98(40): 1-4. 